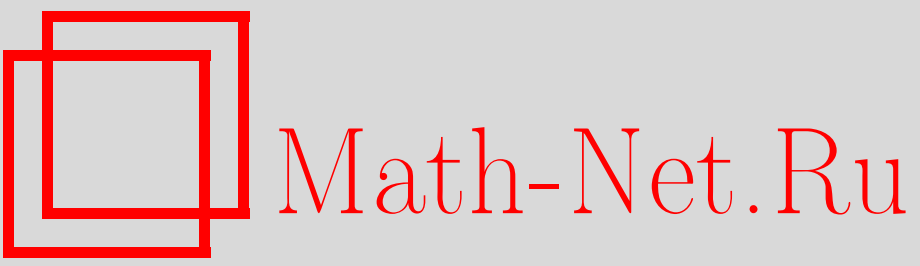

Е. Зеррик, А. Эль Кабусс, Р. Лариши, Оптимальное управление для бесконечномерных гиперболических билинейных систем, Итоги науки и техн. Сер. Соврем. мат. и ее прил. Темат. обз., 2020, том 178, 77-90

DOI: https://doi.org/10.36535/0233-6723-2020-178-77-90

Использование Общероссийского математического портала Math-Net.Ru подразумевает, что вы прочитали и согласны с пользовательским соглашением

http: //www. mathnet.ru/rus/agreement

Параметры загрузки:

IP : 34.229 .108 .108

26 апреля 2023 г., 15:59:00 


\title{
ОПТИМАЛЬНОЕ УПРАВЛЕНИЕ ДЛЯ БЕСКОНЕЧНОМЕРНЫХ ГИПЕРБОЛИЧЕСКИХ БИЛИНЕЙНЫХ СИСТЕМ
}

\author{
(c) 2020 г. $\quad$ Е. ЗЕРРИК, А. ЭЛЬ КАБУСС, Р. ЛАРИШИ
}

\begin{abstract}
АннотАция. Целью настоящей работы является изучение задачи регионального оптимального управления для класса бесконечномерных гиперболических билинейных систем, эволюционирующих в пространственной области $\Omega$. В работе предложен метод построения оптимального управления, доставляющего минимум функционала, который задает невязку между желаемым и конечным состоянием. Достоверность метода подтверждается результатами компьютерного моделирования.
\end{abstract}

Ключевые слова: гиперболическая система, бесконечномерная билинейная система, распределенное оптимальное управление, региональная управляемость.

\section{OUTPUT OPTIMAL CONTROL OF INFINITE-DIMENSIONAL HYPERBOLIC BILINEAR SYSTEMS}

\author{
(c) 2020 E. ZERRIK, A. EL KABOUSS, R. LARHRISSI
}

\begin{abstract}
In this paper, we examine a regional optimal control problem for a class of infinitedimensional hyperbolic bilinear systems evolving on a spatial domain $\Omega$. We characterize an optimal control that minimizes a cost functional, which is composed of the gap between the desired state and final state using optimality conditions. The approach is successfully illustrated by simulations.
\end{abstract}

Keywords and phrases: hyperbolic system, infinite-dimensional bilinear system, distributed optimal control, regional controllability.

AMS Subject Classification: 93D15

1. Введение. Многие физические, химические и биологические явления могут быть эффективно смоделированы бесконечномерными билинейными системами, зависящими от произведения управления и переменных состояния. Такие системы возникают, например, при описании ядерной цепной реакции (см. [11]) или роста аваскулярной опухоли (см. [9]).

Управляемость билинейных систем исследовалась во многих работах. В [2] было показано, что множество достижимости из исходного состояния имеет плотное дополнение в пространстве состояний. Это делает сложным нахождение точного решения задачи управления. В [4] доказано, что точная управляемость зависит от выбора функциональных пространств, и с помощью теории моментов и теоремы Нэша-Мозера показал управляемость для билинейного уравнения линии прогиба балки.

В предыдущих работах исследовалась управляемость конкретных гиперболических билинейных систем. В [12] охарактеризовано оптимальное управление для билинейного волнового уравнения, минимизирующее невязку между желаемым и конечным состоянием, в случае, когда желаемое состояние имеет вид $\left(z_{d}, 0\right)$. Однако этот подход не может быть применен в случае желаемого 
состояния с ненулевой скоростью. Приближенная управляемость для одномерного билинейного волнового уравнения с условиями Неймана была рассмотрена в [3]. В [11] показано, что приближенная управляемость для одномерного билинейного волнового уравнения может быть получена в классе билинейных управлений, не зависящих от времени. В [13] этот результат был обобщен на многомерный случай.

Концепция региональной управляемости для эволюционирующих в пространственной области $\Omega$ бесконечномерных систем, разработанная в [6], относится к изучению классического понятия управляемости только на подобласти $\omega \subset \Omega$. Основные причины для рассмотрения этого понятия заключаются в том, что оно близко к реальным приложениям, например, к моделированию туннельной печи, когда необходимо поддерживать заданную температуру только в ее части. Кроме того, существуют системы, управляемые на некоторой подобласти, но не управляемые во всем пространстве состояний. Региональная управляемость изучалась для параболических линейных систем (см. [7]), затем для гиперболических линейных систем в случае внутренней подобласти (см. [17]) и граничной подобласти (см. [18]). На основе анализа оптимальной системы (см. [19]) и на основе решения уравнения Риккати (см. [20]) эти результаты были расширены до случая параболических билинейных систем с квадратичным функционалом.

Задача регионального оптимального управления для билинейных систем с параболическим оператором четвертого порядка с ограниченным и неограниченным управлением была рассмотрена в [15]. Позднее в [16] исследован класс бесконечномерных билинейных систем в обоих случаях неограниченного и ограниченного управления.

В данной работе рассматривается задача регионального оптимального управления для бесконечномерных гиперболических билинейных систем с распределенным неограниченным управлением. Приводится характеристика оптимального управления и исследуется его единственность. Найденное управление позволяет минимизировать невязку между конечным и желаемым состоянием, имеющим вид $\left(z_{1}^{d}, z_{2}^{d}\right)$. Таким образом, работа является расширением работы [12].

Более точно, пусть $\Omega$ - открытая ограниченная область $\mathbb{R}^{n}$ с регулярной границей $\partial \Omega$. Для $T>0$ введем обозначения $Q=\Omega \times] 0, T[, \Sigma=\partial \Omega \times] 0, T[$ и рассмотрим систему

$$
\begin{cases}\frac{\partial^{2} z(x, t)}{\partial t^{2}}+A z(x, t)=u(x, t) B z(x, t) & \text { в } Q, \\ z(x, 0)=z_{0}(x), \frac{\partial z}{\partial t}(x, 0)=z_{1}(x) & \text { в } \Omega, \\ z(x, t)=0 & \text { на } \Sigma,\end{cases}
$$

где $A$ - самосопряженный дифференциальный оператор второго порядка, имеющий вид

$$
A z=-\sum_{i, j=1}^{n} \frac{\partial}{\partial x_{i}}\left(a_{i j}(x) \frac{\partial z}{\partial x_{j}}\right)+\sum_{i=1}^{n} b_{i}(x) \frac{\partial z}{\partial x_{i}}+c(x) z,
$$

где $a_{i j}=a_{j i}, b_{i}, c \in C^{1}(\Omega)$, и существует такое $\alpha>0$, что

$$
\sum_{i, j=1}^{n} a_{i j} \varepsilon_{i} \varepsilon_{j} \geqslant \alpha|\varepsilon|^{2} \quad \forall \varepsilon=\left(\varepsilon_{1}, \varepsilon_{2}, \ldots, \varepsilon_{n}\right) \in \mathbb{R}^{n} .
$$

Здесь $B: L^{2}(\Omega) \longrightarrow L^{\infty}(\Omega)$ является линейным ограниченным самосопряженным оператором, и множество управлений есть $U=L^{2}(Q)$.

Рассмотрим гильбертово пространство $W=H_{0}^{1}(\Omega) \times L^{2}(\Omega)$ со скалярным произведением

$$
\left\langle\left(f_{1}, f_{2}\right),\left(g_{1}, g_{2}\right)\right\rangle_{W}=\left\langle A^{1 / 2} f_{1}, A^{1 / 2} g_{1}\right\rangle_{L^{2}(\Omega)}+\left\langle f_{2}, g_{2}\right\rangle_{L^{2}(\Omega)} .
$$

Для $\left(z_{0}, z_{1}\right) \in W$ и $u \in U$ система (1.1) имеет единственное решение, для которого $(z, \partial z / \partial t) \in$ $C([0, T], W)($ см. [14]). 
Пусть $\omega$-открытая подобласть области $\Omega, \chi_{\omega}: L^{2}(\Omega) \rightarrow L^{2}(\omega)$-оператор сужения на $\omega$ и $\chi_{\omega}^{*}: L^{2}(\omega) \rightarrow L^{2}(\Omega)$ - оператор, сопряженный к $\chi_{\omega}:$

$$
\chi_{\omega}^{*} z(x, \cdot)= \begin{cases}z(x, \cdot), & \text { если } x \in \omega, \\ 0 & \text { если } x \in \Omega \backslash \omega .\end{cases}
$$

Пусть $z_{d}=\left(z_{1}^{d}, z_{2}^{d}\right) \in L^{2}(\omega) \times L^{2}(\omega)$ - желаемое состояние.

Рассматривается следующая задача оптимального управления:

$$
\min _{u \in U} J(u)
$$

где

$$
J(u)=\frac{1}{2}\left\|\chi_{\omega} z(x, t)-z_{1}^{d}(x)\right\|_{L^{2}\left(0, T ; L^{2}(\omega)\right)}^{2}+\frac{1}{2}\left\|\chi_{\omega} \frac{\partial z(x, T)}{\partial t}-z_{2}^{d}(x)\right\|_{L^{2}(\omega)}^{2}+\frac{\beta}{2}\|u\|_{L^{2}\left(0, T ; L^{2}(\Omega)\right)}^{2},
$$

где $\beta$ - положительная константа.

Цель данной работы - охарактеризовать оптимальное управление в задаче (1.3). Работа организована следующим образом. В разделе 2 доказано существование оптимального управления для задачи (1.3). В разделе 3 найдено оптимальное управление и исследована его единственность. В разделе 4 предложен численный метод поиска оптимального управления, приводящий к алгоритму, который проиллюстрирован посредством компьютерного моделирования.

2. Существование оптимального управления. Данный раздел посвящен доказательству существования оптимального управления в задаче (1.3).

Лемма 1. Рассмотрим следуюшую систему:

$$
\begin{cases}\frac{\partial^{2} z(x, t)}{\partial t^{2}}+A z(x, t)=u(x, t) B z(x, t)+f(x, t) & \text { в } Q, \\ z(x, 0)=z_{0}(x), \frac{\partial z}{\partial t}(x, 0)=z_{1}(x) & \text { в } \Omega, \\ z(x, t)=0 & \text { на } \Sigma,\end{cases}
$$

где $f \in L^{2}\left(0, T ; L^{2}(\Omega)\right)$. Решение $(z, \partial z / \partial t)$ системь (2.1) удовлетворяет следующим неравенствам:

$$
\begin{aligned}
& \sup _{0 \leqslant t \leqslant T}\left(\|z(t)\|_{H_{0}^{1}(\Omega)}+\left\|\frac{\partial z(t)}{\partial t}\right\|_{L^{2}(\Omega)}\right) \leqslant C\left(\left\|z_{0}\right\|_{H_{0}^{1}(\Omega)}+\left\|z_{1}\right\|_{L^{2}(\Omega)}+\|f\|_{L^{2}\left(0, T ; L^{2}(\Omega)\right)}\right), \\
& \left\|\frac{\partial^{2} z_{\ell}(t)}{\partial t^{2}}\right\|_{L^{2}\left(0, T ; H^{-1}(\Omega)\right)} \leqslant C\left(\left\|z_{0}\right\|_{H_{0}^{1}(\Omega)}+\left\|z_{1}\right\|_{L^{2}(\Omega)}+\|u\|_{L^{2}\left(0, T ; L^{2}(\Omega)\right)}+\|f\|_{L^{2}\left(0, T ; L^{2}(\Omega)\right)}\right) .
\end{aligned}
$$

Доказательство. Пусть $\left(w_{m}\right)_{m \geqslant 1}$ задает ортонормированный базис в $L^{2}(\Omega)$ и ортогональный базис в $H_{0}^{1}(\Omega)$. Для положительной константы $\ell$ положим

$$
z_{\ell}(x, t):=\sum_{m=1}^{\ell} d_{\ell}^{m}(t) w_{m}(x)
$$

где $d_{\ell}^{m}(t)(0 \leqslant t \leqslant T, m=1,2, \ldots, \ell)$ удовлетворяют условиям

$$
d_{\ell}^{m}(0)=\left\langle z_{0}, w_{m}\right\rangle_{L^{2}(\Omega)}, \quad \frac{\partial d_{\ell}^{m}(0)}{\partial t}=\left\langle z_{1}, w_{m}\right\rangle_{L^{2}(\Omega)} .
$$

Из системы (2.1) получаем

$$
\left\langle\frac{\partial^{2} z_{\ell}}{\partial t^{2}}, w_{m}\right\rangle_{L^{2}(\Omega)}+\left\langle A z_{\ell}, w_{m}\right\rangle_{L^{2}(\Omega)}=\left\langle u B z_{\ell}, w_{m}\right\rangle_{L^{2}(\Omega)}+\left\langle f, w_{m}\right\rangle_{L^{2}(\Omega)} .
$$


Умножая (2.5) на $\partial d_{\ell}^{m}(t) / \partial t$, суммируя по $m=1$ до $\ell$ и используя $(2.4)$, находим

$$
\left\langle\frac{\partial^{2} z_{\ell}}{\partial t^{2}}, \frac{\partial z_{\ell}}{\partial t}\right\rangle_{L^{2}(\Omega)}+\left\langle A z_{\ell}, \frac{\partial z_{\ell}}{\partial t}\right\rangle_{L^{2}(\Omega)}=\left\langle u B z_{\ell}, \frac{\partial z_{\ell}}{\partial t}\right\rangle_{L^{2}(\Omega)}+\left\langle f, \frac{\partial z_{\ell}}{\partial t}\right\rangle_{L^{2}(\Omega)} .
$$

Так как

$$
\left\langle A z_{\ell}, \frac{\partial z_{\ell}}{\partial t}\right\rangle_{L^{2}(\Omega)}=\frac{1}{2} \frac{\partial}{\partial t}\left\langle A^{1 / 2} z_{\ell}, A^{1 / 2} z_{\ell}\right\rangle_{L^{2}(\Omega)}
$$

имеем

$$
\frac{1}{2} \frac{\partial}{\partial t}\left(\left\|\frac{\partial z_{\ell}}{\partial t}\right\|_{L^{2}(\Omega)}^{2}+\left\|z_{\ell}\right\|_{H_{0}^{1}(\Omega)}^{2}\right) \leqslant\left\|u B z_{\ell}\right\|_{L^{2}(\Omega)}\left\|\frac{\partial z_{\ell}}{\partial t}\right\|_{L^{2}(\Omega)}+\|f\|_{L^{2}(\Omega)}\left\|\frac{\partial z_{\ell}}{\partial t}\right\|_{L^{2}(\Omega)} .
$$

Поскольку оператор $B$ ограничен, заключаем, что

$$
\frac{\partial}{\partial t}\left(\left\|\frac{\partial z_{\ell}}{\partial t}\right\|_{L^{2}(\Omega)}^{2}+\left\|z_{\ell}\right\|_{H_{0}^{1}(\Omega)}^{2}\right) \leqslant C\left(\|u\|_{L^{2}(\Omega)}\left(\left\|z_{\ell}\right\|_{H_{0}^{1}(\Omega)}^{2}+\left\|\frac{\partial z_{\ell}}{\partial t}\right\|_{L^{2}(\Omega)}^{2}\right)+\|f\|_{L^{2}(\Omega)}^{2}\right) .
$$

Введем обозначение

$$
\Phi(t):=\left\|\frac{\partial z_{\ell}}{\partial t}\right\|_{L^{2}(\Omega)}^{2}+\left\|z_{\ell}\right\|_{H_{0}^{1}(\Omega)}^{2} ;
$$

тогда неравенство (2.7) принимает вид

$$
\frac{\partial \Phi(t)}{\partial t} \leqslant C\|u\|_{L^{2}(\Omega)} \Phi(t)+C\|f(t)\|_{L^{2}(\Omega)}^{2} .
$$

В силу неравенства Гронуолла имеем

$$
\Phi(t) \leqslant e^{\left(C_{1}\|u\|_{L^{2}(\Omega)}\right) t}\left(\Phi(0)+C_{2} \int_{0}^{t}\|f(s)\|_{L^{2}(\Omega)}^{2} d s\right) .
$$

Тогда

$$
\left(\left\|z_{\ell}(t)\right\|_{H_{0}^{1}(\Omega)}+\left\|\frac{\partial z_{\ell}(t)}{\partial t}\right\|_{L^{2}(\Omega)}\right) \leqslant C\left(\left\|z_{0}\right\|_{H_{0}^{1}(\Omega)}+\left\|z_{1}\right\|_{L^{2}(\Omega)}+\|f\|_{L^{2}\left(0, T ; L^{2}(\Omega)\right)}\right) .
$$

Теперь, используя $(2.5)$, для $y \in H_{0}^{1}(\Omega)$, удовлетворяющего условию $\|y\|_{H_{0}^{1}(\Omega)} \leqslant 1$, получаем

Таким образом,

$$
\left|\left\langle\frac{\partial^{2} z_{\ell}}{\partial t}, y\right\rangle\right| \leqslant C\left(\|f\|_{L^{2}(\Omega)}+\|u\|_{L^{2}(\Omega)}\left\|z_{\ell}\right\|_{H_{0}^{1}(\Omega)}\right) .
$$

$$
\int_{0}^{T}\left\|\frac{\partial^{2} z_{\ell}}{\partial t^{2}}\right\|_{H^{-1}(\Omega)}^{2} d t \leqslant C\left(\|f\|_{L^{2}\left(0, T ; L^{2}(\Omega)\right)}^{2}+\left\|z_{0}\right\|_{H_{0}^{1}(\Omega)}^{2}+\left\|z_{1}\right\|_{L^{2}(\Omega)}^{2}+\|u\|_{L^{2}\left(0, T ; L^{2}(\Omega)\right)}^{2}\right) .
$$

При $\ell \rightarrow+\infty$ получаем (2.2) и (2.3).

Теорема 1. Существует оптимальное управление $u \in U$, решающее задачу (1.3).

Доказательство. Выполним те же шаги, что и в [12, теорема 2.1], используя неравенства (2.2) и (2.3) для доказательства слабой сходимости $z_{u_{m}}, \partial z_{u_{m}} / \partial t$ и $*$-слабой сходимости $\partial^{2} z_{u_{m}} / \partial t^{2}$ для минимизирующей последовательности $u_{m}$. Тогда, используя результат о компактности (см. [10]), получаем сильную сходимость $z_{u_{m}} \rightarrow z_{u^{*}}$ в $L^{\infty}\left(0, T, L^{2}(\Omega)\right)$. Это дает слабую сходимость последовательности $u_{m} B z_{u_{m}}$. При $m \rightarrow \infty$ заключаем, что $\left(z_{u^{*}}, \partial z_{u^{*}} / \partial t\right)$ является решением системы (1.1) под действием управления $u^{*}$. Используя непрерывность оператора и полунепрерывность $J$ снизу относительно слабой сходимости, получаем

$$
J\left(u^{*}\right) \leqslant \lim _{m \rightarrow \infty} \inf J\left(u_{m}\right)=I
$$

значит, $u^{*}$ является оптимальным управлением. 
3. Характеристика и единственность оптимального управления. В этом разделе дана характеристика оптимального управления в задаче (1.3) и обсуждается вопрос единственности. Сначала докажем следующую лемму.

Лемма 2. Отображение $\Theta: u \in U \rightarrow\left(z_{u}, \partial z_{u} / \partial t\right) \in C(0, T, W)$ дифферениируемо по Фреше, его производная удовлетворяет системе

$$
\begin{cases}\frac{\partial^{2} y}{\partial t^{2}}+A y=u(x, t) B y+h(x, t) B z & \text { в } Q, \\ y(x, 0)=\frac{\partial y}{\partial t}(x, 0)=0 & \text { в } \Omega, \\ y(x, t)=0 & \text { на } \Sigma .\end{cases}
$$

Доказательство. Отображение $L: h \in U \rightarrow(y, \partial y / \partial t) \in C([0, T], W)$ линейно. Используя неравенство (2.2), получаем

$$
\left\|\left(y, \frac{\partial y}{\partial t}\right)\right\|_{C(0, T, W)} \leqslant C\|h\|_{U} .
$$

Следовательно, $L$ непрерывно. Положим

$$
\left(z_{h+u}, \frac{\partial z_{h+u}}{\partial t}\right)=\Theta(u+h), \quad\left(\psi, \frac{\partial \psi}{\partial t}\right)=\left(z_{h+u}-z, \frac{\partial}{\partial t}\left(z_{h+u}-z\right)\right) .
$$

Тогда $(\psi, \partial \psi / \partial t)$ является слабым решением системы

$$
\begin{cases}\frac{\partial^{2} \psi}{\partial t^{2}}+A \psi=u(x, t) B \psi+h(x, t) B z_{h+u} & \text { в } Q, \\ \psi(x, 0)=\frac{\partial \psi}{\partial t}(x, 0)=0 & \text { в } \Omega, \\ \psi(x, t)=0 & \text { на } \Sigma .\end{cases}
$$

Таким образом,

$$
\left\|\left(\psi, \frac{\partial \psi}{\partial t}\right)\right\|_{C(0, T, W)} \leqslant C\|h\|_{U} .
$$

Теперь, если

$$
\left(\varphi, \frac{\partial \varphi}{\partial t}\right)=\left(\psi-y, \frac{\partial}{\partial t}(\psi-y)\right)
$$

то $(\varphi, \partial \varphi / \partial t)$ является слабым решением следующей системы:

$$
\begin{cases}\frac{\partial^{2} \varphi}{\partial t^{2}}+A \varphi=u(x, t) B \varphi+h(x, t) B \psi & \text { в } Q, \\ \varphi(x, 0)=\frac{\partial \varphi}{\partial t}(x, 0)=0 & \text { в } \Omega, \\ \varphi(x, t)=0 & \text { на } \Sigma .\end{cases}
$$

Заключаем, что

$$
\left\|\left(\varphi, \frac{\partial \varphi}{\partial t}\right)\right\|_{C(0, T ; W)} \leqslant C\|h\|_{U}^{2}
$$

где $(\varphi, \partial \varphi / \partial t)=\Theta(u+h)-\Theta(u)-\Theta^{\prime}(u) \cdot h$, что завершает доказательство.

Теорема 2. Оптимальное управление $u^{*} \in U$ в задаче (1.3) имеет вид

$$
u^{*}(x, t)=-\frac{1}{\beta} B z(x, t) p(x, t)
$$




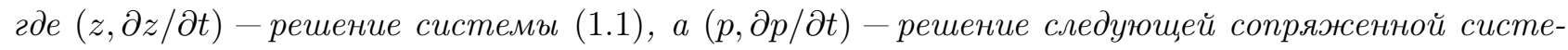
Mbl:

$$
\begin{cases}\frac{\partial^{2} p(x, t)}{\partial t^{2}}+A p(x, t)=B u(x, t) p(x, t)+z(x, t)-\chi_{\omega}^{*} z_{1}^{d}(x) & \text { в } Q, \\ p(x, T)=\left(\frac{\partial z(x, T)}{\partial t}-\chi_{\omega}^{*} z_{2}^{d}(x)\right) & в \Omega, \\ \frac{\partial p(x, T)}{\partial t}=0 & \text { в } \Omega, \\ p(x, t)=0 & \text { на } \Sigma .\end{cases}
$$

Доказательство. Вычислим производную функционала $J$ относительно $u$ по направлению $h$. Дифференцируемость отображения $\Theta$ и его нормы гарантирует дифференцируемость $J$. Производная имеет вид

$$
\begin{aligned}
& J^{\prime}(u) \cdot h=\int_{\Omega} \chi_{\omega}^{*} \chi_{\omega} \int_{0}^{T} y(x, t)\left(z(x, t)-\chi_{\omega}^{*} z_{1}^{d}(x)\right) d t d x+ \\
& \quad+\int_{\Omega} \chi_{\omega}^{*} \chi_{\omega} \frac{\partial y(x, T)}{\partial t}\left(\frac{\partial z(x, T)}{\partial t}-\chi_{\omega}^{*} z_{2}^{d}(x)\right) d x+\beta \int_{Q} h(x, t) u(x, t) d t d x .
\end{aligned}
$$

В силу системы (3.3) имеем

$$
\begin{aligned}
J^{\prime}(u) \cdot h=\int_{\Omega} \chi_{\omega}^{*} \chi_{\omega} \int_{0}^{T} y(x, t)(z(x, t) & \left.-\chi_{\omega}^{*} z_{1}^{d}(x)\right) d t d x+ \\
& +\int_{\Omega} \chi_{\omega}^{*} \chi_{\omega} \frac{\partial y(x, T)}{\partial t} p(x, T) d x+\beta \int_{Q} h(x, t) u(x, t) d t d x .
\end{aligned}
$$

Интегрируя по частям, в силу системы (3.3) получаем

$$
\begin{aligned}
J^{\prime}(u) \cdot h= & \int_{\Omega} \chi_{\omega}^{*} \chi_{\omega} \int_{0}^{T} y(x, t)\left(\frac{\partial^{2} p(x, t)}{\partial t^{2}}+A p(x, t)-B u(x, t) p(x, t)\right) d t d x+ \\
& +\int_{\Omega} \chi_{\omega}^{*} \chi_{\omega} \int_{0}^{T}\left(\frac{\partial^{2} y(x, t)}{\partial t^{2}} p(x, t)+\frac{\partial y(x, t)}{\partial t} \frac{\partial p(x, t)}{\partial t}\right) d t d x+\beta \int_{Q} h(x, t) u(x, t) d t d x .
\end{aligned}
$$

Повторное интегрирование по частям дает

$$
\begin{aligned}
& J^{\prime}(u) \cdot h=\int_{\Omega} \chi_{\omega}^{*} \chi_{\omega}\left[y(x, t) \frac{\partial p(x, t)}{\partial t}\right]_{0}^{T} d x- \\
&-\int_{\Omega} \chi_{\omega}^{*} \chi_{\omega} \int_{0}^{T} \frac{\partial y(x, t)}{\partial t} \frac{\partial p(x, t)}{\partial t} d t d x+\int_{\Omega} \chi_{\omega}^{*} \chi_{\omega} \int_{0}^{T} y(x, t)(A p(x, t)-B u(x, t) p(x, t)) d t d x+ \\
& \quad+\int_{0}^{T} \chi_{\omega}^{*} \chi_{\omega} \int_{\Omega}\left(\frac{\partial^{2} y(x, t)}{\partial t^{2}} p(x, t)+\frac{\partial y(x, t)}{\partial t} \frac{\partial p(x, t)}{\partial t}\right) d x d t+\beta \int_{Q} h(x, t) u(x, t) d t d x .
\end{aligned}
$$


Следовательно,

$$
\begin{aligned}
J^{\prime}(u) \cdot h=\int_{\Omega} \chi_{\omega}^{*} \chi_{\omega} \int_{0}^{T}(A y(x, t) & -u(x, t) B y(x, t)) p(x, t) d t d x+ \\
& +\int_{0}^{T} \chi_{\omega}^{*} \chi_{\omega} \int_{\Omega} \frac{\partial^{2} y(x, t)}{\partial t^{2}} p(x, t) d x d t+\beta \int_{Q} h(x, t) u(x, t) d t d x .
\end{aligned}
$$

Система (3.1) влечет

$$
\begin{aligned}
J^{\prime}(u) \cdot h=\int_{\Omega} \int_{0}^{T} h(x, t) B z(x, t) \chi_{\omega}^{*} \chi_{\omega} p(x, t) d t d x & +\beta \int_{Q} h u d t d x= \\
& =\int_{Q} h\left(\beta u+B z(x, t) \chi_{\omega}^{*} \chi_{\omega} p(x, t) d x\right) d t d x .
\end{aligned}
$$

Таким образом, условие $J^{\prime}(u) \cdot h=0$ дает

$$
u^{*}(x, t)=-\frac{1}{\beta} B z(x, t) \chi_{\omega}^{*} \chi_{\omega} p(x, t)
$$

Следствие 1. При условии, что $u^{*}(x, t)=v^{*}(t) \mathbb{1}_{D}(x)$, где $D \subset \Omega$-область приложения управления, а $\mathbb{1}_{D}$ - характеристическая функиия с ненулевой мерой $\mu(D)$, оптимальное управление $v^{*}(t)$ имеет вид

$$
v^{*}(t)=-\frac{1}{\beta \mu(D)} \int_{\Omega} B z(x, t) \chi_{\omega}^{*} \chi_{\omega} p(x, t) d x .
$$

Оптимальное управление $v^{*}$ зависит от области $\omega$ и области приложсения управления $D$.

Замечание 1. Заметим, что для задачи (1.3) со множеством управлений $U_{M}=\left\{u \in L^{\infty}(Q) \mid\right.$ $-M \leqslant u \leqslant M\}$, где $M$ - заданная константа, то $J^{\prime}(u) \cdot h \geqslant 0$, что означает

$$
\int_{Q} h\left(\beta u+B z(x, t) \chi_{\omega}^{*} \chi_{\omega} p(x, t)\right) d t d x \geqslant 0 .
$$

Используя границы множества управлений, получаем

$$
u^{*}(x, t)=\max \left(-M, \min \left(-\frac{1}{\beta} B z(x, t) \chi_{\omega}^{*} \chi_{\omega} p(x, t), M\right)\right) .
$$

В предположении, что $u^{*}(x, t)=v^{*}(t) \mathbb{1}_{D}(x)$, получаем, что оптимальное управление $v^{*}(t)$ выражается следующим образом:

$$
v^{*}(t)=\max \left(-M, \min \left(-\frac{1}{\beta \mu(D)} \int_{\Omega} B z(x, t) \chi_{\omega}^{*} \chi_{\omega} p(x, t) d x, M\right)\right) .
$$

Замечание 2. В случае $A=-\Delta, B=I$ и $\omega=\Omega$ управление (3.5)) минимизирует невязку между желаемым состоянием $\left(z_{1}^{d}, z_{2}^{d}\right)$ и конечным состоянием $\left(z_{u}, \partial z_{u} / \partial t\right)$ - решением билинейного волнового уравнения. Заметим, что управление, найденное в [12], неприменимо в этом случае.

Приведем далее достаточные условия единственности оптимального управления.

Теорема 3. Предположим, что решения систем (1.1) и (3.3) ограничены на Q. При достаточно малом Т задача (1.3) имеет единственное решение. 
Доказательство. Предположим, что существуют два решения $u_{1}, u_{2}$ задачи (1.3). Пусть $\left(\tilde{z}_{1}, \partial \tilde{z}_{1} / \partial t\right),\left(\tilde{z}_{2}, \partial \tilde{z}_{2} / \partial t\right)$ - соответствующие состояния и $\left(\tilde{p}_{1}, \partial \tilde{p}_{1} / \partial t\right),\left(\tilde{p}_{2}, \partial \tilde{p}_{2} / \partial t\right)$ - соответствующие решения сопряженной системы под действием управлений $u_{1}$ и $u_{2}$.

Пусть $\lambda>0, \bar{z}_{1}=e^{-\lambda t} \tilde{z}_{1}, \bar{z}_{2}=e^{-\lambda t} \tilde{z}_{2}, \bar{p}_{1}=e^{\lambda t} \tilde{p}_{1}$ и $\bar{p}_{2}=e^{\lambda t} \tilde{p}_{2} ;\left(\bar{z}_{j}, \frac{\partial \bar{z}_{j}}{\partial t}\right)$ для $j=1,2$ удовлетворяют следующей системе:

$$
\begin{cases}\frac{\partial^{2} \bar{z}_{j}(x, t)}{\partial t^{2}}+A \bar{z}_{j}(x, t)+2 \lambda \frac{\partial \bar{z}_{j}}{\partial t}(x, t)+\lambda^{2} \bar{z}_{j}(x, t)=u_{j} B \bar{z}_{j}(x, t) & \text { в } Q, \\ \bar{z}_{j}(x, 0)=z_{0}(x) & \text { в } \Omega, \\ \frac{\partial \bar{z}_{j}}{\partial t}(x, 0)=z_{1}(x) & \text { в } \Omega, \\ \tilde{z}(x, t)=0 & \text { на } \Sigma .\end{cases}
$$

С другой стороны, $\left(\bar{z}_{1}-\bar{z}_{2}\right)$ удовлетворяет следующей системе:

$$
\begin{cases}\frac{\partial^{2}\left(\bar{z}_{1}-\bar{z}_{2}\right)}{\partial t^{2}}(x, t)+A\left(\bar{z}_{1}-\bar{z}_{2}\right)(x, t)+2 \lambda \frac{\partial\left(\bar{z}_{1}-\bar{z}_{2}\right)}{\partial t}(x, t)+\lambda^{2}\left(\bar{z}_{1}-\bar{z}_{2}\right)(x, t)+ & \\ \quad=u_{1} B\left(\bar{z}_{1}-\bar{z}_{2}\right)(x, t)+\left(u_{1}-u_{2}\right) B \bar{z}_{2}(x, t) & \text { в } Q, \\ \left(\bar{z}_{1}-\bar{z}_{2}\right)(x, 0)=\frac{\partial\left(\bar{z}_{1}-\bar{z}_{2}\right)}{\partial t}(x, 0)=0 & \text { в } \Omega, \\ \left(\bar{z}_{1}-\bar{z}_{2}\right)(x, t)=0 & \text { на } \Sigma .\end{cases}
$$

Умножая уравнение в (3.7) на $\partial\left(\bar{z}_{1}-\bar{z}_{2}\right) / \partial t$ и интегрируя по области $Q$, получаем

$$
\begin{aligned}
& \int_{0}^{T}\left\langle\left\langle\frac{\partial^{2}\left(\bar{z}_{1}-\bar{z}_{2}\right)}{\partial t^{2}}, \frac{\partial\left(\bar{z}_{1}-\bar{z}_{2}\right)}{\partial t}\right\rangle_{L^{2}(\Omega)}+\left\langle A\left(\bar{z}_{1}-\bar{z}_{2}\right), \frac{\partial\left(\bar{z}_{1}-\bar{z}_{2}\right)}{\partial t}\right\rangle_{L^{2}(\Omega)}+\right. \\
& \left.+2 \lambda\left\langle\frac{\partial\left(\bar{z}_{1}-\bar{z}_{2}\right)}{\partial t}, \frac{\partial\left(\bar{z}_{1}-\bar{z}_{2}\right)}{\partial t}\right\rangle_{L^{2}(\Omega)}+\lambda^{2}\left\langle\left(\bar{z}_{1}-\bar{z}_{2}\right), \frac{\partial\left(\bar{z}_{1}-\bar{z}_{2}\right)}{\partial t}\right\rangle_{L^{2}(\Omega)}\right) d t= \\
& =\int_{0}^{T}\left(\left\langle u_{1} B\left(\bar{z}_{1}-\bar{z}_{2}\right), \frac{\partial\left(\bar{z}_{1}-\bar{z}_{2}\right)}{\partial t}\right\rangle_{L^{2}(\Omega)}+\left\langle\left(u_{1}-u_{2}\right) B \bar{z}_{2}, \frac{\partial\left(\bar{z}_{1}-\bar{z}_{2}\right)}{\partial t}\right\rangle_{L^{2}(\Omega)}\right) d t .
\end{aligned}
$$

Интегрирование по частям дает следующее выражение:

$$
\begin{aligned}
& \left\|\frac{1}{2} \frac{\partial\left(\bar{z}_{1}-\bar{z}_{2}\right)}{\partial t}(x, T)\right\|_{L^{2}(\Omega)}^{2}+\frac{1}{2}\left\|A^{1 / 2}\left(\bar{z}_{1}-\bar{z}_{2}\right)(x, T)\right\|_{L^{2}(\Omega)}^{2}+ \\
& +\frac{\lambda^{2}}{2}\left\|\left(\bar{z}_{1}-\bar{z}_{2}\right)(x, T)\right\|_{L^{2}(\Omega)}^{2}+2 \lambda \int_{0}^{T}\left\|\frac{\partial\left(\bar{z}_{1}-\bar{z}_{2}\right)}{\partial t}\right\|_{L^{2}(\Omega)}^{2} d t= \\
& =\int_{0}^{T}\left(\left\langle u_{1} B\left(\bar{z}_{1}-\bar{z}_{2}\right), \frac{\partial\left(\bar{z}_{1}-\bar{z}_{2}\right)}{\partial t}\right\rangle_{L^{2}(\Omega)}+\left\langle\left(u_{1}-u_{2}\right) B \bar{z}_{2}, \frac{\partial\left(\bar{z}_{1}-\bar{z}_{2}\right)}{\partial t}\right\rangle_{L^{2}(\Omega)}\right) d t .
\end{aligned}
$$

Используя неравенство

$$
\begin{aligned}
\left\|u_{1}-u_{2}\right\|_{L^{2}(\Omega)}=\left\|B \bar{z}_{1} \chi_{\omega}^{*} \chi_{\omega} \bar{p}_{1}-B \bar{z}_{2} \chi_{\omega}^{*} \chi_{\omega} \bar{p}_{2}\right\|_{L^{2}(\Omega)} & \leqslant \\
& \leqslant\left\|B\left(\bar{z}_{1}-\bar{z}_{2}\right) \chi_{\omega}^{*} \chi_{\omega} \bar{p}_{2}-B \tilde{z}_{1} \chi_{\omega}^{*} \chi_{\omega}\left(\bar{p}_{1}-\bar{p}_{2}\right)\right\|_{L^{2}(\Omega)},
\end{aligned}
$$


получаем

$$
2 \lambda \int_{0}^{T}\left\|\frac{\partial\left(\bar{z}_{1}-\bar{z}_{2}\right)}{\partial t}\right\|_{L^{2}(\Omega)}^{2} d t \leqslant C_{1} \int_{0}^{T}\left(\left\|\left(\bar{z}_{1}-\bar{z}_{2}\right)\right\|_{L^{2}(\Omega)}^{2}+\left\|\frac{\partial\left(\bar{z}_{1}-\bar{z}_{2}\right)}{\partial t}\right\|_{L^{2}(\Omega)}^{2}+\left\|\left(\bar{p}_{1}-\bar{p}_{2}\right)\right\|_{L^{2}(\Omega)}^{2}\right) d t
$$

где $C_{1}$ зависит от $B, \bar{z}_{1}, \bar{z}_{2}, \bar{p}_{1}$ и $\bar{p}_{2}$.

С другой стороны, $\left(\bar{p}_{1}-\bar{p}_{2}\right)$ является решением следующей системы:

$$
\begin{cases}\frac{\partial^{2}\left(\bar{p}_{1}-\bar{p}_{2}\right)}{\partial t^{2}}(x, t)+A\left(\bar{p}_{1}-\bar{p}_{2}\right)(x, t)-2 \lambda\left(\bar{p}_{1}-\bar{p}_{2}\right)= & \\ \quad=B u_{1}\left(\bar{p}_{1}-\bar{p}_{2}\right)(x, t)+B\left(u_{1}-u_{2}\right) \bar{p}_{2}(x, t)+e^{\lambda t}\left(\bar{z}_{1}-\bar{z}_{2}\right)(x, t) & \text { в } Q, \\ \left(\bar{p}_{1}-\bar{p}_{2}\right)(x, T)=\lambda e^{2 \lambda T}\left(\bar{z}_{1}-\bar{z}_{2}\right)(x, T)+e^{2 \lambda T} \frac{\partial\left(\bar{z}_{1}-\bar{z}_{2}\right)}{\partial t}(x, T) & \text { в } \Omega, \\ \frac{\partial \bar{p}(x, T)}{\partial t}=0 & \text { в } \Omega, \\ \left(\bar{p}_{1}-\bar{p}_{2}\right)(x, t)=0 & \text { на } \Sigma .\end{cases}
$$

Умножая уравнение в $(3.11)$ на $\partial\left(\bar{p}_{1}-\bar{p}_{2}\right) / \partial t$, интегрируя по области $Q$ и используя $(3.9)$, получаем

$$
\begin{aligned}
2 \lambda \int_{0}^{T}\left\|\frac{\partial\left(\bar{p}_{1}-\bar{p}_{2}\right)}{\partial t}\right\|_{L^{2}(\Omega)}^{2} d t \leqslant & \\
\leqslant C_{2} \int_{0}^{T}\left(\left\|\left(\bar{z}_{1}-\bar{z}_{2}\right)\right\|_{L^{2}(\Omega)}^{2}+\right. & \left.\left\|\frac{\partial\left(\bar{z}_{1}-\bar{z}_{2}\right)}{\partial t}\right\|_{L^{2}(\Omega)}^{2}+\left\|\frac{\partial\left(\bar{p}_{1}-\bar{p}_{2}\right)}{\partial t}\right\|_{L^{2}(\Omega)}^{2}+\left\|\left(\bar{p}_{1}-\bar{p}_{2}\right)\right\|_{L^{2}(\Omega)}^{2}\right) d t+ \\
& +\frac{\lambda^{2} e^{4 \lambda T}}{2}\left\|\left(\bar{z}_{1}-\bar{z}_{2}\right)(x, T)\right\|_{L^{2}(\Omega)}^{2}+\frac{e^{4 \lambda T}}{2}\left\|\frac{\partial\left(\bar{z}_{1}-\bar{z}_{2}\right)}{\partial t}(x, T)\right\|_{L^{2}(\Omega)}^{2} .
\end{aligned}
$$

Используя (3.8), получаем

$$
\begin{array}{r}
2 \lambda \int_{0}^{T}\left\|\frac{\partial\left(\bar{p}_{1}-\bar{p}_{2}\right)}{\partial t}\right\|_{L^{2}(\Omega)}^{2} d t \leqslant C_{3} e^{4 \lambda T} \int_{0}^{T}\left(\left\|\left(\bar{z}_{1}-\bar{z}_{2}\right)\right\|_{L^{2}(\Omega)}^{2}+\left\|\frac{\partial\left(\bar{z}_{1}-\bar{z}_{2}\right)}{\partial t}\right\|_{L^{2}(\Omega)}^{2}+\right. \\
\left.+\left\|\frac{\partial\left(\bar{p}_{1}-\bar{p}_{2}\right)}{\partial t}\right\|_{L^{2}(\Omega)}^{2}+\left\|\left(\bar{p}_{1}-\bar{p}_{2}\right)\right\|_{L^{2}(\Omega)}^{2}\right) d t .
\end{array}
$$

Складывая неравенства (3.10) и (3.12), приходим к следующему неравенству:

$$
\begin{gathered}
2 \lambda \int_{0}^{T}\left(\left\|\frac{\partial\left(\bar{p}_{1}-\bar{p}_{2}\right)}{\partial t}\right\|_{L^{2}(\Omega)}^{2}+\left\|\frac{\partial\left(\bar{z}_{1}-\bar{z}_{2}\right)}{\partial t}\right\|_{L^{2}(\Omega)}^{2}\right) d t \leqslant\left(C_{1}+C_{3} e^{4 \lambda T}\right) \int_{0}^{T}\left(\left\|\left(\bar{z}_{1}-\bar{z}_{2}\right)\right\|_{L^{2}(\Omega)}^{2}+\right. \\
\left.+\left\|\frac{\partial\left(\bar{z}_{1}-\bar{z}_{2}\right)}{\partial t}\right\|_{L^{2}(\Omega)}^{2}+\left\|\frac{\partial\left(\bar{p}_{1}-\bar{p}_{2}\right)}{\partial t}\right\|_{L^{2}(\Omega)}^{2}+\left\|\left(\bar{p}_{1}-\bar{p}_{2}\right)\right\|_{L^{2}(\Omega)}^{2}\right) d t .
\end{gathered}
$$


Далее

$$
\begin{gathered}
\int_{0}^{T}\left\|\left(\bar{z}_{1}-\bar{z}_{2}\right)\right\|_{L^{2}(\Omega)}^{2} d t=\int_{0}^{T} \int_{\Omega}\left(\int_{0}^{t} \frac{\partial\left(\bar{z}_{1}-\bar{z}_{2}\right)}{\partial s} d s\right)^{2} d x d t \leqslant \\
\leqslant \int_{0}^{T} \int_{\Omega} t\left(\int_{0}^{t}\left(\frac{\partial\left(\bar{z}_{1}-\bar{z}_{2}\right)}{\partial s}\right)^{2} d s\right) d x d t \leqslant \frac{T^{2}}{2} \int_{0}^{T} \int_{\Omega}\left(\frac{\partial\left(\bar{z}_{1}-\bar{z}_{2}\right)}{\partial t}\right)^{2} d x d t \leqslant \\
\leqslant \frac{T^{2}}{2} \int_{0}^{T}\left\|\frac{\partial\left(\bar{z}_{1}-\bar{z}_{2}\right)}{\partial t}\right\|_{L^{2}(\Omega)}^{2} d t
\end{gathered}
$$

и

$$
\int_{0}^{T}\left\|\left(\bar{p}_{1}-\bar{p}_{2}\right)\right\|_{L^{2}(\Omega)}^{2} d t \leqslant \frac{T^{2}}{2} \int_{0}^{T}\left\|\frac{\partial\left(\bar{p}_{1}-\bar{p}_{2}\right)}{\partial t}\right\|_{L^{2}(\Omega)}^{2} d t .
$$

Для фиксированного $\lambda>0$ и достаточно малого $T$, для которых

получаем

$$
2 \lambda>\left(C_{1}+C_{3} e^{4 \lambda T}\right)\left(\frac{T^{2}}{2}+1\right)
$$

а значит,

$$
\int_{0}^{T}\left\|\frac{\partial\left(\bar{z}_{1}-\bar{z}_{2}\right)}{\partial t}\right\|_{L^{2}(\Omega)}^{2} d t+\int_{0}^{T}\left\|\frac{\partial\left(\bar{p}_{1}-\bar{p}_{2}\right)}{\partial t}\right\|_{L^{2}(\Omega)}^{2} d t=0,
$$

что дает $\bar{z}_{1}=\bar{z}_{2}, \bar{p}_{1}=\bar{p}_{2}$.

$$
\frac{\partial\left(\bar{p}_{1}-\bar{p}_{2}\right)}{\partial t}=\frac{\partial\left(\bar{z}_{1}-\bar{z}_{2}\right)}{\partial t}=0
$$

4. Алгоритм и компьютерное моделирование. В этом разделе предлагается алгоритм для вычисления оптимального управления в задаче (1.3).

В случае неограниченных управлений оптимальное управление может быть рассчитано по формуле

$$
\left\{\begin{array}{l}
u_{m+1}=-\frac{1}{\beta} B z_{u_{m}}(x, t) \chi_{\omega}^{*} \chi_{\omega} p_{u_{m}}(x, t) \\
u_{1}=0
\end{array}\right.
$$

а в случае ограниченных управлений - по формуле

$$
\left\{\begin{array}{l}
u_{m+1}=\max \left(-M, \min \left(-\frac{1}{\beta} B z_{u_{m}}(x, t) \chi_{\omega}^{*} \chi_{\omega} p_{u_{m}}(x, t), M\right)\right), \\
u_{1}=0
\end{array}\right.
$$

где $z_{u_{m}}$ - решение системы (1.1), а $p_{u_{m}}$ - решение сопряженной системы $(3.3)$, соответствующие управлению $u_{m}$.

\section{Алгоритм 1.}

Шаг 1: Исходные данные.

(i) Исходное состояние $\left(z_{0}, z_{1}\right)$, подобласть $\omega$ и желаемое состояние $\left(z_{1}^{d}, z_{2}^{d}\right)$.

(ii) Положительная константа $\beta$, момент времени $T$, пороговая точность $\varepsilon$ и $u_{1}=0$.

Шаг 2: Повторять, пока выполняется неравенство $\left\|u_{m+1}-u_{m}\right\|_{U} \leqslant \varepsilon$.

(i) Найти $z_{u_{m}}$, решая систему (1.1) методом конечных разностей.

(ii) Найти $p_{u_{m}}$, решая систему (3.3) методом конечных разностей.

(iii) Вычислить $u_{m+1}$ по формуле (4.1) в случае неограниченных управлений или по формуле (4.2) в случае ограниченных управлений.

Шаг 3: Найденное управление $u_{m}$ - решение задачи (1.3). 
Компьютерное моделирование. Положим $\Omega=] 0,1[$. Рассмотрим билинейную систему с волновым уравнением

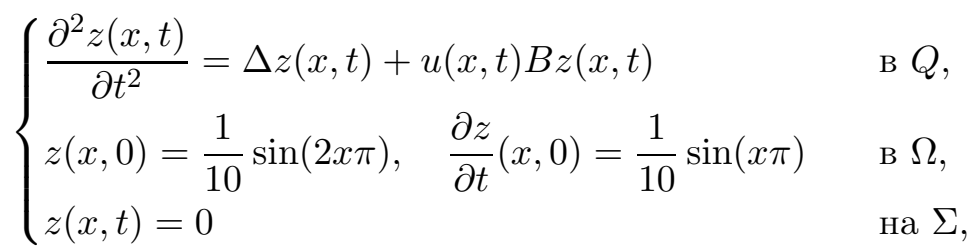

где $T=2$,

$$
B z(x, t)=\int_{\Omega} z(x, t) d x, \quad u(x, t)=v(t) \mathbb{1}_{D}
$$

и $D=] 0,32 ; 0,8[$ - область приложения управления.

Пример 1. Пусть $\omega \subset \Omega$, рассмотрим систему (4.3) и задачу (1.3) при $z_{1}^{d}=0, z_{2}^{d}=0$ и $\beta=0,45$.

Случай ограниченных управлений: $U=\left\{u \in L^{\infty}(Q) \mid-0,015 \leqslant u \leqslant 0,015\right\}$. Применяя предложенный алгоритм с

$$
v^{*}(t)=\max \left(-M, \min \left(-\frac{1}{\beta \mu(D)} \int_{\Omega} B z(x, t) \chi_{\omega}^{*} \chi_{\omega} p(x, t) d x, M\right)\right),
$$

получаем следующие результаты для различных подобластей.

1. Для $\omega=] 0 ; 0,3\left[\right.$, график $v^{*}$ представлен на рис. 1(а), а достигнутое состояние и скорость на рис. $1(\mathrm{~b})$ и $1(\mathrm{c})$ соответственно. Желаемое состояние на $\omega$ достигнуто с точностью $\rho=$ $1,42 \times 10^{-5}$ и значением функционала $J\left(v^{*}\right)=5,47 \times 10^{-1}$.

2. Для $\omega=] 0,6 ; 0,9\left[\right.$, график $v^{*}$ представлен на рис. 2(a), а достигнутое состояние и скорость на рис. $2(\mathrm{~b})$ и $2(\mathrm{c})$ соответственно. Желаемое состояние на $\omega$ достигнуто с точностью $\rho=$ $7,29 \times 10^{-5}$ и значением функционала $J\left(v^{*}\right)=6,73 \times 10^{-1}$.

Пример 2. Пусть $\omega \subset \Omega$, рассмотрим систему (4.3) и задачу (1.3) при

$$
z_{1}^{d}=-\frac{1}{10} \sin (x \pi) \sin (x \pi), \quad z_{2}^{d}=-\frac{1}{10} \sin (x \pi) \cos (2 x \pi), \quad \beta=0,45 .
$$

Случай неограниченных управлений: $U=L^{2}(Q)$. Применяя предложенный алгоритм с

$$
\varepsilon=10^{-4}, \quad v(t)=-\frac{1}{\beta \mu(D)} \int_{\Omega} B z(x, t) \chi_{\omega}^{*} \chi_{\omega} p(x, t) d x,
$$

получаем следующие результаты для различных подобластей.

1. Для $\omega=] 0 ; 0,3\left[\right.$ график $v^{*}$ представлен на рис. 3(a), а достигнутое состояние и скорость - на pис. 3(b) и 3(c) соответственно. Желаемое состояние на $\omega$ достигнуто с точностью

$$
\rho=\left\|z(x, T)-z_{1}^{d}(x)\right\|_{L^{2}(\omega)}^{2}+\left\|\frac{\partial z}{\partial t}(x, T)-z_{2}^{d}(x)\right\|_{L^{2}(\omega)}^{2}=2,46 \times 10^{-4}
$$

и значением функционала $J\left(v^{*}\right)=0,0891$.

2. Для $\omega=] 0,6 ; 0,9\left[\right.$, график $v^{*}$ представлен на рис. $4(\mathrm{a})$, , а достигнутое состояние и скорость на рис. $4(\mathrm{~b})$ и $4(\mathrm{c})$ соответственно. Желаемое состояние на $\omega$ достигнуто с точностью $\rho=$ $8,71 \times 10^{-4}$ и значением функционала $J\left(v^{*}\right)=0,3591$.

5. Заключение. Рассмотрена задача регионального оптимального управления для бесконечномерных гиперболических билинейных систем. Предложен метод построения оптимального управления, доставляющего минимум функционала, который задает невязку между желаемым и конечным состоянием на заданной подобласти пространства состояний. Полученные результаты успешно протестированы с помощью численных экспериментов. Случай граничной подобласти остается открытым. 


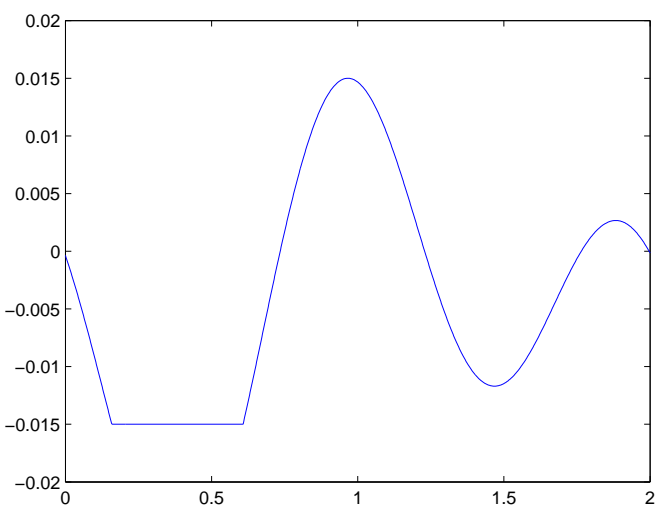

(a)

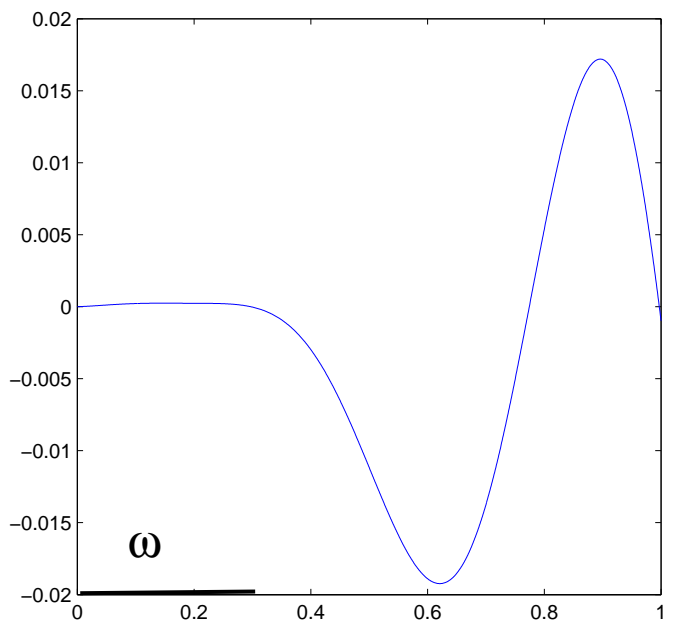

(b)

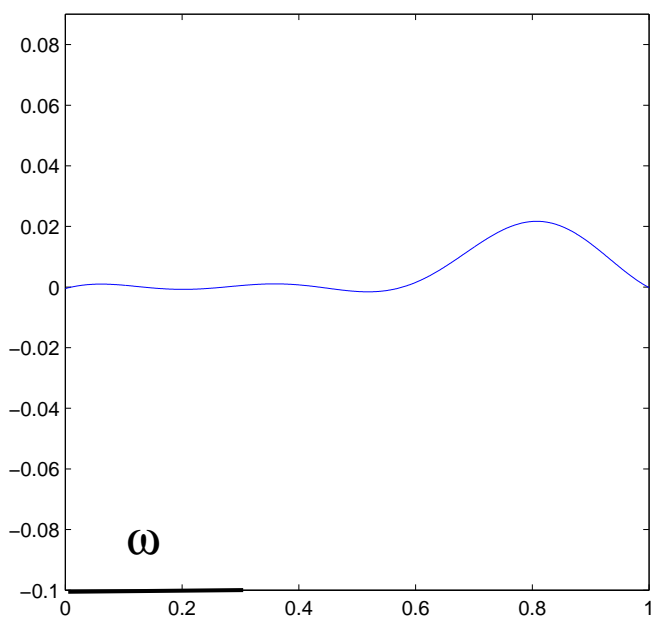

(c)

Рис. 1

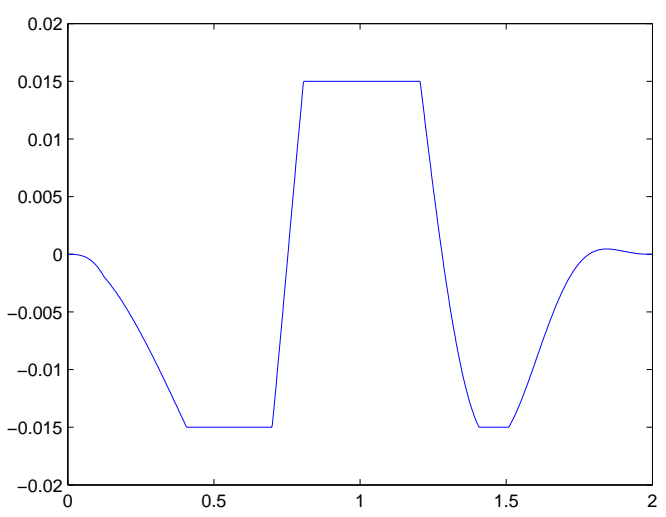

(a)

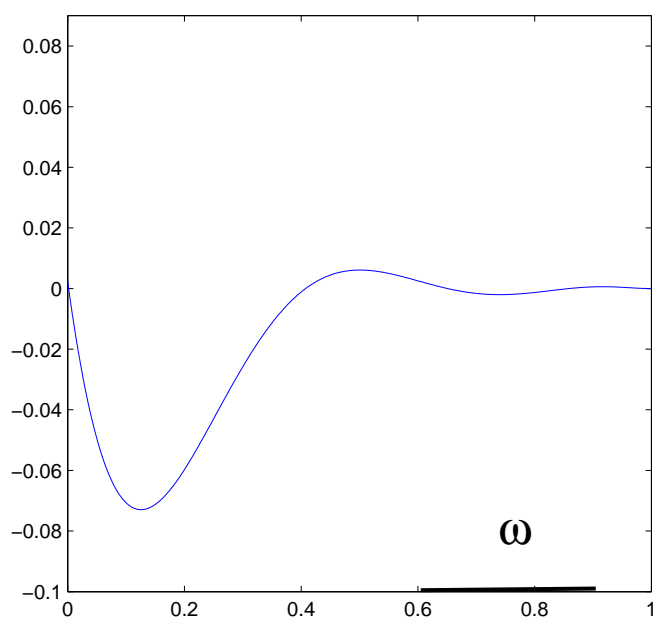

(b)

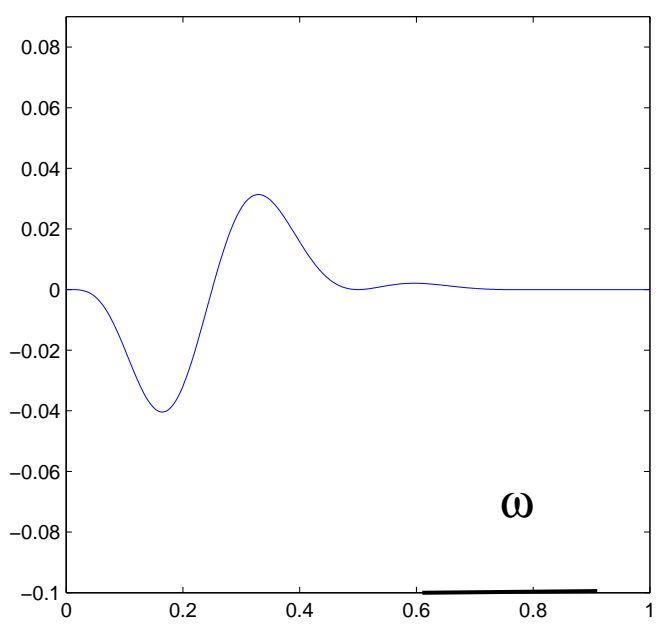

(c)

Рис. 2 


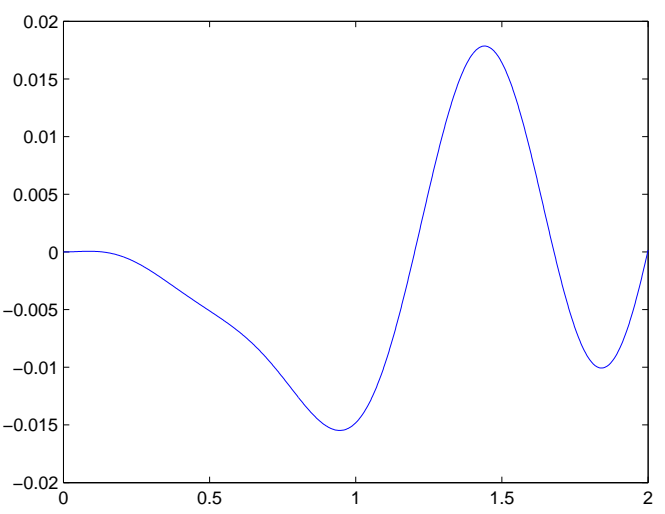

(a)

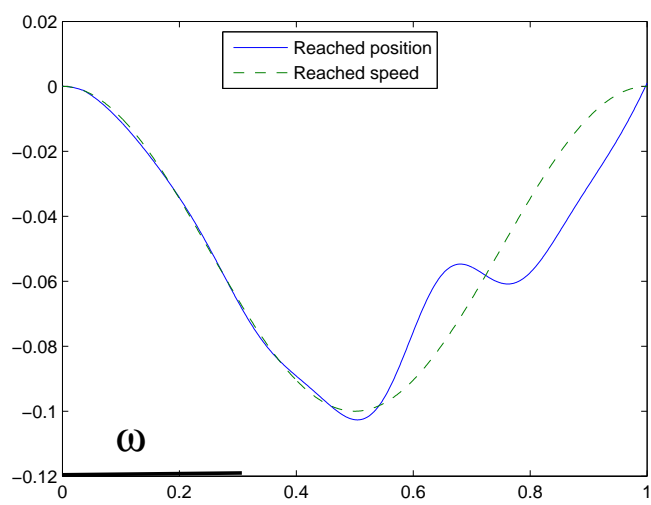

(b)

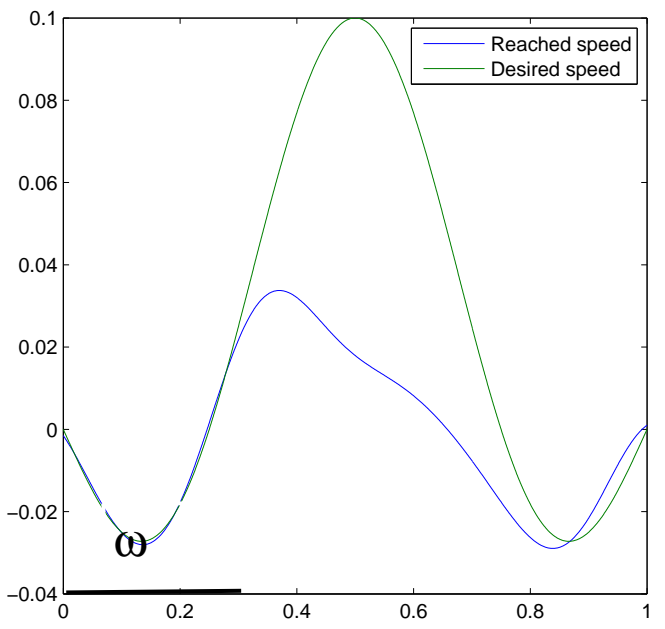

(c)

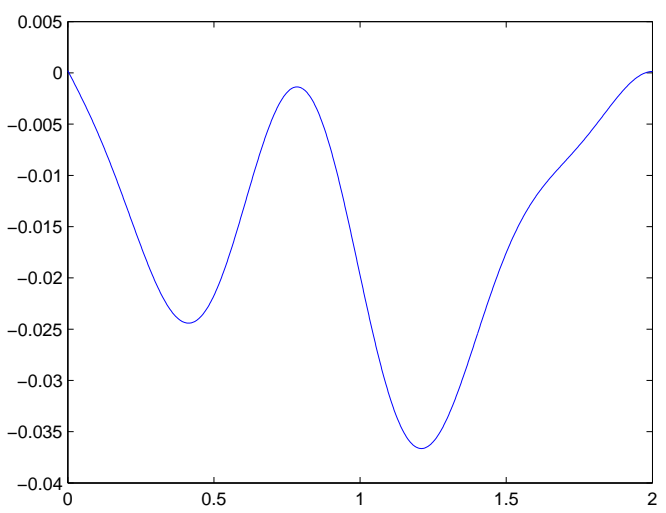

(a)

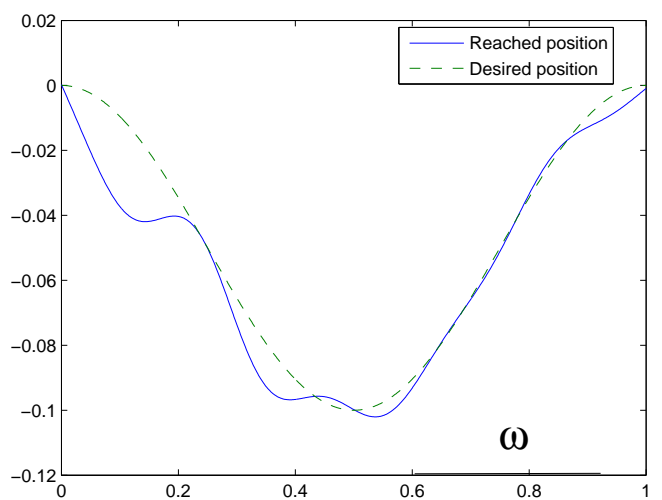

(b)

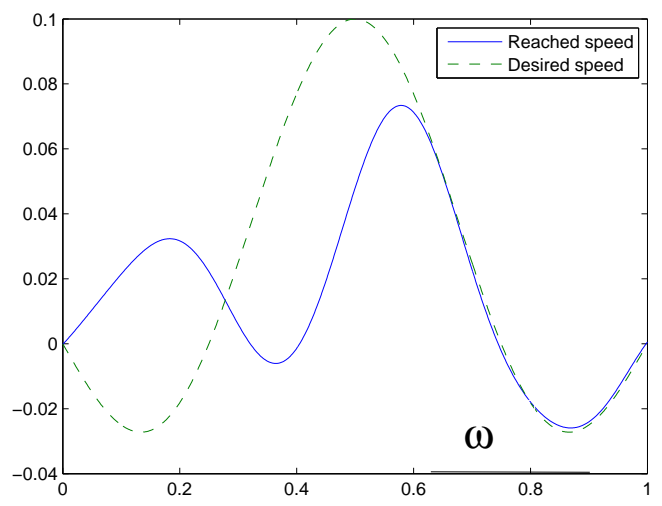

(c)

Рис. 3

Рис. 4 


\section{СПИСОК ЛИТЕРАТУРЫ}

1. Adams R. A. Sobolev Spaces. - New York-San Francisco-London: Academic Press, 1975.

2. Ball J. M., Marsden J. E. Slemrod M. Controllability for distributed bilinear systems// SIAM J. Control Optim. - 1982. - 20, № 4. - P. 575-597.

3. Beauchard K. Local controllability and noncontrollability for a $1 \mathrm{~d}$ wave equation with bilinear control// J. Differ. Equations. — 2011. — 250, № 4. - P. 2064-2098.

4. Beauchard K. Local controllability of a one-dimensional beam equation// SIAM J. Control Optim. - 2008 . — 47, № 3. - P. 1219-1273.

5. Brezis H. Analyse Fonctionnelle. Théorie et Applications. — Paris: Masson, 1983.

6. El Jai A., Zerrik E., Afiri L. Systems Theory : Modeling, Analysis and Control. — Presse Univ. Perpignan, 2009.

7. El Jai A., Zerrik E., Pritchard A. Regional controllability of distributed parameter systems// Int. J. Control. - 1995. - 62, № 6. - P. 1351-1365.

8. Evans L. C. Partial Differential Equations. - Providence, Rhode Island: Am. Math. Soc., 1998.

9. Ferreira S. C. Jr., Martins M. L., Vilela M. J. A reaction-diffusion model for the growth of avascular tumor// Phys. Rev. — 2002. - 65, № 2. - 021907 .

10. Jacques $S$. Compact sets in the space $L^{p}(0, T ; B) / /$ Ann. Mat. Pura Appl. — 1987. — 146, № 6. — P. 65-96.

11. Khapalov A. Y. Controllability of Partial Differential Equations Governed by Multiplicative Controls. Springer-Verlag, 2010.

12. Liang M. Bilinear optimal control for a wave equation// Math. Models Meth. Appl. Sci. — 1999. — 9, № 1. - P. 45-68.

13. Ouzahra M. Controllability of the wave equation with bilinear controls// Eur. J. Control. - 2014. - 20, № 2. - P. 57-63.

14. Pazy A. Semigroups of Linear Operators and Applications to Partial Differential Equations. - BerlinHeidelberg-New York-Tokyo: Springer-Verlag, 1983.

15. Zerrik E., El Kabouss A. Regional optimal control of a class of bilinear systems// IMA J. Math. Control Inform. - 2017. - 34, № 4. - P. 1157-1175.

16. Zerrik E., El Kabouss A. Regional optimal control of a class of infinite-dimensional bilinear systems// Int. J. Control. — 2017. — 90, № 7. - P. 1495-1504.

17. Zerrik E., Larhrissi R. Regional target control of the wave equation// Int. J. Syst. Sci. - 2001. - 32, № 10. - P. 1233-1242.

18. Zerrik E., Larhrissi R. Regional boundary controllability of hyperbolic systems. Numerical approach// J. Dynam. Control syst. - 2002. - 8, № 3. - P. 293-311.

19. Zerrik E., Ould sidi M. "Regional controllability for infinite-dimensional bilinear systems: Approach and simulations// Int. J. Control. — 2011. - 84, № 12. - P. 2108-2116.

20. Ztot K., Zerrik E., Bourray H. Regional control problem for distributed bilinear systems: Approach and simulations// Int. J. Appl. Math. Comput. Sci. — 2011. — 21, № 3. - P. 499-580.

Zerrik E.

Университет Мулая Исмаила, Мекнесс, Марокко

E-mail: zerrik3@yahoo.fr

El Kabouss A.

Университет Мулая Исмаила, Мекнесс, Марокко

Larhrissi R.

Университет Мулая Исмаила, Мекнесс, Марокко

E-mail: r.larhrissi@gmail.com 